\title{
Diffuse Gastric Cancer with Peritoneal Carcinomatosis Can Mimic Crohn's Disease
}

\author{
C. Hommel ${ }^{a} \quad$ M. Knoedler ${ }^{b} \quad$ C. Bojarski ${ }^{c} \quad$ M. Schumann ${ }^{c}$ \\ H.J. Epple ${ }^{c} \quad$ M. Zeitz ${ }^{c} \quad$ S. Daum ${ }^{c}$ \\ ${ }^{a}$ Université Catholique de Louvain, Bruxelles, Belgium; ${ }^{b}$ Department of \\ Hematology and Oncology and 'Medical Clinic I Gastroenterology, Infectious \\ Diseases, Rheumatology, Charité - Campus Benjamin Franklin, Berlin, Germany
}

\section{Key Words}

Diffuse gastric cancer - Linitis plastica - Peritoneal carcinomatosis - Crohn's disease · Endoscopy Endoscopic ultrasound

\begin{abstract}
In some cases the diagnosis of gastric cancer is difficult and the endoscopic presentation may be misleading. Diffuse type gastric carcinoma with peritoneal metastasis may present primarily with abdominal pain, colonic infiltration and/or diarrhea, thus other differential diagnoses like Crohn's disease (CD) may be considered at first. Therefore intensive diagnostic work-up is important. We report two cases of gastric cancer with ascites due to peritoneal carcinomatosis who were first diagnosed as CD. The patients were hospitalized in different institutions for weight loss, abdominal pain and nausea. The first colonoscopy, upper endoscopy with multiple biopsies and ascites puncture were negative for malignant disease, but macroscopic lesions resembling CD were described. Both patients were released on a prednisolone-based treatment for suspected CD. They presented to our hospital for further evaluation due to persistent symptoms. Neither lower nor upper endoscopy were suggestive of $C D$ and endoscopic ultrasound was suspicious of malignancy in one case. Histology was diagnostic and showed gastric infiltration by a poorly differentiated adenocarcinoma. Diffuse type gastric cancer (gastric linitis plastica) with peritoneal metastasis may mimic certain clinical, endoscopic and CT imaging features of CD. Repeated biopsies and endoscopic investigations are often necessary to confirm a malignant process, especially in case of an inconclusive clinical and endoscopic picture. Endoscopic ultrasound may be useful to evaluate the risk of malignancy in patients with macroscopic suspicion of malignancy and negative biopsies.
\end{abstract}




\section{Introduction}

The prevalence of Crohn's disease (CD) in North America ranges from 26.0 to 198.5 cases per 100,000 persons [1]. Clinical symptoms as well as endoscopic aspects of $\mathrm{CD}$ are nonspecific, even if some lesions can be highly suggestive. Diffuse gastric adenocarcinoma with peritoneal carcinomatosis (PC) is much less frequent but can mimic certain aspects of CD [2,3]. Gastric adenocarcinomas, which represent about $85 \%$ of gastric malignant tumors, may be subdivided into two categories: diffuse type, in which cell cohesion is absent so that individual cells infiltrate and thicken the stomach wall without forming a discrete mass, and intestinal type, characterized by cohesive neoplastic cells that form glandular-like tubular structures. Diffuse carcinomas occur more often in younger patients, develop throughout the stomach, result in a loss of elasticity of the gastric wall (so-called linitis plastica or 'leather bottle' appearance) and carry a poorer prognosis. Studies also show that PC has a stronger association with this type of cancer (frequency of PC in patients with poor histology against other subtypes: 40 vs. $13 \%$; $p=0.0001$, relative risk 3.07) [4], whereas visceral metastases are more frequent with the intestinal type. In contrast to intestinal type carcinomas, which tend to predominate in high-risk geographic regions, the incidence of diffuse carcinomas is similar in most populations [5].

We present two clinical cases initially diagnosed as CD, but were later on revealed to be suffering from diffuse gastric cancer with PC. We stress out similarities which can exist between the presentation of a patient with $\mathrm{CD}$ and a patient with malignant disease. These cases show that repeated diagnostic evaluation with multilevel biopsies is essential in case of unconfirmed CD in order to confirm or dismiss this hypothesis.

\section{Case 1}

A 44-year-old Caucasian male was hospitalized in our institution for further diagnostics and evaluation of suspected CD. The patient had presented to a different institution 1 month earlier with a history of recurrent upper abdominal cramps, accompanied by a $10 \mathrm{~kg}$ weight loss over a period of 6 months. He also complained about nausea and occasional bilious vomiting. There was no change in stool frequency or consistency, no fever or night sweats. He was a smoker (17 pack years) and denied alcohol abuse. He had never had any surgery or been seriously ill before. Family history revealed that his father had died of an undefined cancer at the age of 56 years. He was taking no medication. Laboratory testing in the external hospital revealed minor normochromic, normocytic anemia (Hb: $13.5 \mathrm{~g} / \mathrm{dl}$, normal: 14-17.5). Inflammatory parameters were normal. Tumor markers were not determined. Upper endoscopy showed mucosal edema with luminal narrowing in the duodenal bulb and gastric antrum. Biopsies showed low-grade chronic inflammation, no signs of malignancy and were positive for Helicobacter pylori. Colonoscopy revealed segmental, superficial, partially ulcerated, about $3 \mathrm{~cm}$ long polypoid lesions in the ascending colon and the transversum. The ileocecal valve was slightly erythematous. Multilevel biopsies showed signs of chronic inactive enteritis, segmental mucosal edema and crypt hyperplasia, but no granulomatous lesions. Multislice CT depicted a small diffuse quantity of ascites as well as segmental concentric wall thickening of the colon. No enlarged lymph nodes were found. Hydro-MRI showed no jejunoileal involvement. Ascites puncture fluid contained elevated cellularity (798/ $\mu \mathrm{l}$, normal: $0-100)$, but no signs of acute inflammation or neoplastic cells. After negative Mendel-Mantoux testing for tuberculosis, the patient was released on a $1 \mathrm{mg} / \mathrm{kg}$ body weight prednisolone therapy, associated with vitamin $\mathrm{D}$ and calcium supplements, for suspicion of inflammatory bowel disease. H. pylori eradication treatment was planned to be started 4 weeks later. Because of his cachectic state hypercaloric nutrition was suggested. 
One month later, the patient presented to our institution with right flank pain and a swollen scrotum. On physical examination he had an altered general appearance and was in a cachectic nutritional status (51 kg/176 cm, BMI $16.4 \mathrm{~kg} / \mathrm{m}^{2}$ ). During abdominal examination he indicated diffuse abdominal tenderness; bowel sounds were normal. Abdominal girth was increased, there was no hepato- or splenomegaly. Scrotal examination revealed increased bilateral girth, no pain or change in skin aspect. No lymphadenopathy was appreciated in the neck, axillae or groin. Further physical examination was normal.

Laboratory testing showed elevated CA19-9 (56 U/ml, normal: <37) (CEA, $\alpha$-FP and PSA were normal), mild hypocalcemia (2.12 mmol/l, normal: 2.15-2.65) and minor normochromic, normocytic anemia (Hb: $13 \mathrm{~g} / \mathrm{dl}$, normal: 14-17.5). Abdominal ultrasound revealed right hydronephrosis, perihepatic, perisplenic and interenteric ascites with a $10 \mathrm{~mm}$ wall thickening of the gastric antrum. Upper endoscopy revealed a lack of gastric motility and difficult insufflation. The antrum, pylorus and duodenal bulb were swollen and deformed with superficial erosions, and colonoscopy showed circular edema ( $\underline{\text { fig. } 1}$ ). The lesser curvature presented with a livid and vulnerable mucosa. Endoscopic ultrasound (EUS) showed thickening of the anterior wall and the lesser curvature up to $10 \mathrm{~mm}$ and extension of the process into the serosa with disruption of individual wall layers (fig. 2). Biopsies taken in the antrum, corpus and fundus showed signs of invasive, poorly (G3) differentiated adenocarcinoma with signet cell infiltration and linitis plastica appearance. Multiple strictures were seen in colonoscopy. Furthermore segmental concentric edemas with glassy wall changes were observed. There were no ulcerations or erosions. Biopsies taken at different levels showed hyperplastic colon mucosa with beginning architectural disturbances. No signs of malignancy were detected in colon biopsies. CT imaging of the abdomen revealed bilateral hydroceles (probably due to venous obstruction by ascitic fluid), right hydronephrosis, wall thickening of the stomach and colon (fig. 3 ) and a high suspicion of PC. A diagnostic ascites puncture confirmed this hypothesis.

The diagnosis of a primary diffuse (Lauren classification) gastric adenocarcinoma cT4N $\times$ M1G3 (stage $\geq$ IIIB) [5], Her-2/neu-negative, with PC was made and FLOT (fluorouracil, leucovorin, oxaliplatin and docetaxel) palliative chemotherapy was started. After 6 cycles of chemotherapy the patient showed a marked clinical response (reduction of initial symptoms) with tumor markers decreasing and undetectable tumor in re-staging thoracoabdominal CT. Due to recurrent ascites, intraperitoneal treatment with the trifunctional anti-EpCAM antibody catumaxomab was started. For the time being, the patient is without any further chemotherapy.

\section{Case 2}

A 58-year-old Caucasian male presented to our institution for further diagnostics and evaluation of suspected CD due to ongoing emesis after meals. The patient had been hospitalized in a different institution 6 weeks earlier with a history of nausea and mild abdominal pain accompanied by a $16 \mathrm{~kg}$ weight loss over the last 5 months.

Stool frequency was slightly increased. The patient had never had any surgery. His family history was uneventful. He was taking no medication. Laboratory testing in the external hospital revealed minor normochromic, normocytic anemia. Inflammatory parameters were normal. Tumor markers were not determined. Upper endoscopy showed signs of Ménétrier disease, but biopsies could not demonstrate malignancy. Colonoscopy revealed segmental stenosis in the ascending colon and several aphthous lesions in the rectum. Biopsies showed unspecific inflammation. The patient was discharged with local-acting budesonide under suspicion of CD.

Due to clinical deterioration, the patient presented to our institution 6 weeks later. On physical examination he had a decreased nutritional status (59 kg/170 cm, BMI $20.4 \mathrm{~kg} / \mathrm{m}^{2}$ ) and showed icteric sclerae. Abdominal examination was uneventful except for signs of ascites.

Laboratory testing showed elevated CA72-4 (150.3 U/ml, normal: <4) and minor normochromic, normocytic anemia (Hb: $13.9 \mathrm{~g} / \mathrm{dl}$, normal: 14-17.5) as well as increased liver enzymes (ALT $277 \mathrm{U} / \mathrm{l}$, normal: <45; AST $154 \mathrm{U} / \mathrm{l}$, normal: <50; direct bilirubin $8.2 \mathrm{mg} / \mathrm{dl}$, normal: $<0.3$ ) with normal liver synthesis parameters. 


\begin{abstract}
Abdominal ultrasound revealed ascites, signs of mechanical cholestasis with slightly dilated peripheral bile ducts but a normal width of the central bile duct. MRI showed gastric and colonic wall thickening, ascites and signs of mechanical cholestasis. Upper endoscopy revealed a swollen distal esophagus without obvious tumor and fluid in the dilated esophagus. Passage of the distal esophagus was only possible with a $5 \mathrm{~mm}$ pediatric endoscope. After passage into the stomach, the gastric folds were swollen without ulcerations or protruding tumor. Passage into the duodenum was not possible due to a narrowed pylorus. Due to the stenotic distal gastroesophageal region, EUS could not be performed. Biopsies taken all over the stomach depicted only chronic inflammation, but the biopsies taken from the gastroesophageal region revealed undifferentiated PAS-positive, Her-2/neu-negative adenocarcinoma with a high proliferation rate of $90 \%$ (Ki67). Colonoscopy confirmed the external finding and biopsies revealed no malignancy as ascites puncture was without detection of malignant cells. Due to the increased liver enzymes including mechanical cholestasis, systemic chemotherapy was introduced using docetaxel and cisplatin without 5-FU in a 50\% reduced dose on a two-weekly rhythm. Following chemotherapy, liver enzymes including bilirubin decreased to normal levels and weight increased to $68 \mathrm{~kg}$ without parenteral nutrition. Re-staging with CT after 3 months showed no more ascites and a marked decrease in the thickening of the gastric as well as the colonic wall without further ascites. After 1 year of systemic therapy, the patient is in good clinical condition and complete remission based on CT findings.
\end{abstract}

\title{
Discussion
}

Although the classical presentation of CD mostly does not cause diagnostic problems, in some cases CD presents with ascites accompanied by only slightly increased stool frequency [6]. When the first patient presented to the hospital, upper endoscopy findings were unspecific. Colonoscopy showed segmental, polypoid, superficial erosions and inflammation of the ileocecal valve with circumferential narrowing. Suspected CD could not be excluded by histopathological findings. One month later we first followed a protocol to confirm a diagnosis of CD and characterize the activity. However, endoscopic aspects of gastric and colonic lesions had evolved and became clearly indicative of a neoplastic process. As biopsies had been negative for malignancy before, EUS presented as a useful diagnostic tool to further evaluate the risk of gastric carcinoma. A study by Ginès et al. [7] demonstrated that the enlargement of deep layers is an independent predictor of malignancy in patients with large gastric folds found in endoscopy without signs of malignancy in gastric biopsies. In this study, the probability of having a malignant process increased from 34 to $94 \%$ in case of thickened deep layers, whereas in the absence of this finding the likelihood decreased from 34 to $4.7 \%$. In both described cases, repeated biopsies confirmed the suspected diagnosis of primary gastric cancer. Distal esophageal stenosis prevented EUS in the second case.

The primary tumor breaching the serosal layer, proof of carcinomatous invasion of the peritoneal cavity by positive cytology and histological findings in gastric biopsies led to the diagnosis of a diffuse gastric adenocarcinoma cT4N $\times$ M1G3. As curative resection (R0) was impossible in this case of $\mathrm{PC}$, the primary standard of care was chemotherapy [8]. Gastric linitis plastica often presents with weight loss, vomiting and/or early satiety and abdominal pain. Lower intestinal symptoms such as diarrhea, constipation or rectal bleeding are less common [9]. In a case of suspected active CD, absent history of diarrhea - a major symptom of CD - should lead to further investigations to rule out or confirm this hypothesis. As predominant upper gastrointestinal tract manifestations are less common, these should initiate additional investigations in unconfirmed cases. 
There have been cases of difficult differential diagnosis between CD and endoscopic lesions. Lee et al. [10] presented the case of a 41-year-old male suffering from abdominal fullness, anorexia and weight loss diagnosed with diffuse type gastric carcinoma in which colonoscopy revealed flat elevated lesions with superficial erosions. In this case biopsies showed signet cell infiltration of the colonic mucosa. Other reports on patients with linitis plastica show colonoscopic findings including granular and friable mucosa, occasional submucosal nodularity (resembling the typical cobblestone appearance of $\mathrm{CD}$ ), superficial erosions, pseudopolyps and luminal narrowing mimicking CD. The first set of biopsies identified malignancy in a minority of these cases [2]. For all strictures in a patient with suspected or confirmed inflammatory bowel disease it is essential to assess evidence of malignancy. The most specific microscopic lesions in $\mathrm{CD}$ are noncaseating granulomas, but these are seen in less than $25 \%$ of mucosal biopsies. Therefore, their absence does not exclude CD. Furthermore, the typical transmural inflammation with monocellular cell infiltrate, fibrosis, ulcers and fissures can be absent in case of inactive CD [9]. At the time of presentation at our institution, rigidity of the gastric wall, a wall thickness exceeding $6 \mathrm{~mm}$, and a poor demarcation between layers seen in EUS, combined with histological findings, proved diffuse gastric cancer described as linitis plastica by endoscopy in our first patient. Linitis plastica with intestinal metastases, PC and CD can present as diffuse concentric wall thickening with segmental stricture pattern on CT. Metastatic polypoid lesions in the intestine usually originate from melanoma or spindle cell renal carcinoma [10], but can also result from poorly differentiated gastric carcinoma [11-13]. In most of these cases repeated deep biopsies are necessary to eventually detect neoplastic cells and firmly diagnose malignancy.

Whereas different other cases of patients with gastric linitis report a certain macroscopic resemblance to $\mathrm{CD}$, the first patient was one of the rarely described cases where colonoscopic appearance in a patient with gastric linitis and PC combined multiple characteristics of CD: skip lesions, strictures and ulcers. The clinical presentation of the two patients was atypical and supported repeated diagnostic evaluation in order to rule out malignancy (fig. 4). Initially this led to a false suspicion which delayed the actual diagnosis and therapy of an aggressive neoplasm.

\section{Conclusion}

The central aim of this report was to illustrate how metastatic diffuse gastric cancer can imitate endoscopic aspects of $\mathrm{CD}$. The clinician should be alarmed by atypical clinical presentations such as a missing history of diarrhea or predominant upper gastrointestinal tract manifestations and constantly be aware that malignant diseases can present in insidious ways. Considering the possible major the rapeutic implications, suspicion of CD should always be confirmed by further diagnostic testing, especially since PC or intestinal metastases of gastric cancer, inter alia, can mimic CD. Repeated biopsies are often necessary to diagnose linitis plastica because of the diffuse character of neoplastic cell infiltration. In case of macroscopic suspicion of gastric malignancy in endoscopy, EUS may be a useful minimally invasive diagnostic tool to further evaluate the patient's risk of gastric cancer. Exact staging of gastric cancer is very important for the selection of patients for whom R0 surgery might be indicated. At this point, palliative chemotherapy is the standard treatment in cases of PC. 
Intraperitoneal application of catumaxomab appears to be a valuable tool to help patients with recurrent ascites and may be a further step in improving outcome of patients with PC due to gastric cancer.

\section{Authors' Contributions}

C.H. carried out the literature review and drafted the manuscript. M.K. critically reviewed the oncologic aspects of the case report. S.M., C.B. and H.J.E. carried out the diagnostic procedures and participated in discussions of the patients' treatment. S.D. designed the case report, participated in the literature review and drafting of the manuscript as well as oncologic follow-up and treatment. M.Z. critically revised the case report and supervised patient care. All authors read and approved the final manuscript.

\section{Disclosure Statement}

The authors declare that they have no conflict of interest.
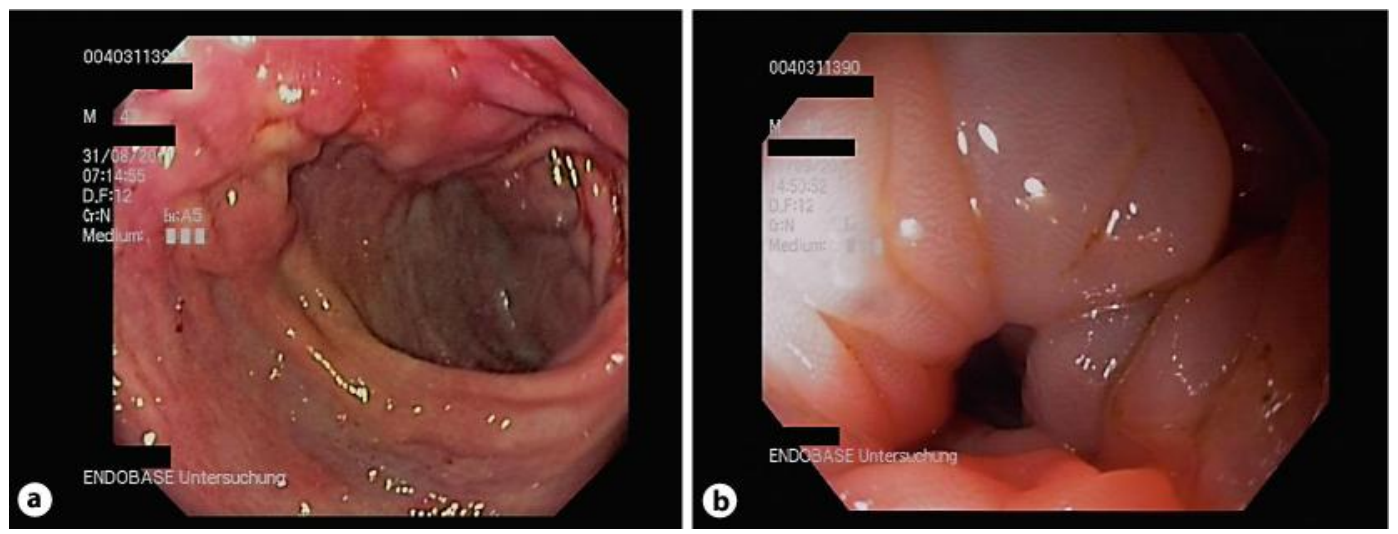

Fig. 1. a Esophagogastroduodenoscopy of the antrum: swollen gastric wall with deformations and superficial erosions. b Colonoscopy: segmental circular edema. 


\begin{tabular}{r|l|l|l}
$\begin{array}{r}\text { Case Reports in } \\
\text { Gastroenterology }\end{array}$ & $\begin{array}{l}\text { Case Rep Gastroenterol 2012;6:695-703 } \\
\text { DOI: 10.1159/000345385 }\end{array}$ & $\begin{array}{l}\text { Published online: } \\
\text { November 7, 2012 }\end{array}$ & $\begin{array}{l}\text { @ 2012 S. Karger AG, Basel } \\
\text { ISSN 1662-0631 } \\
\text { www.karger.com/crg }\end{array}$ \\
\hline
\end{tabular}

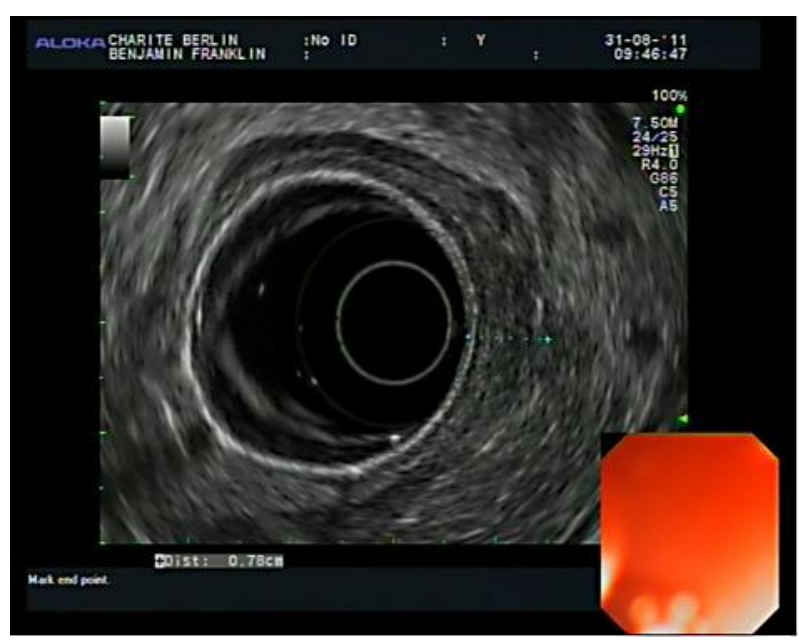

Fig. 2. EUS: thickening of the anterior wall and the lesser curvature up to $10 \mathrm{~mm}$, extension of the process into the serosa and abolished layer differentiation.

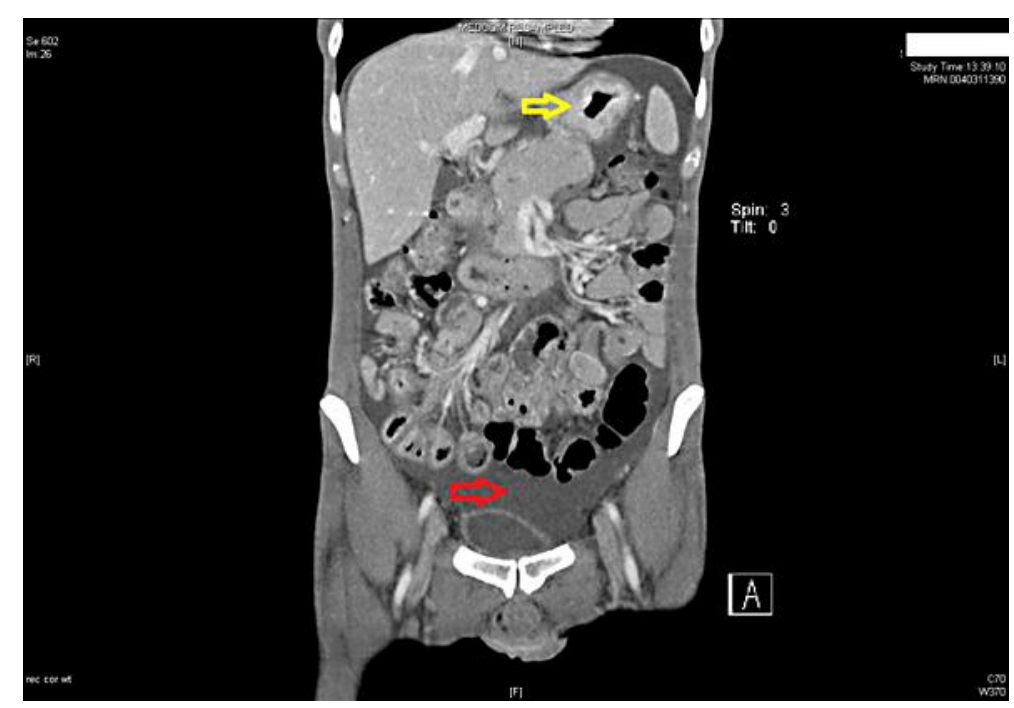

Fig. 3. CT: ascites (red arrow) and gastric wall thickening (yellow arrow). 
-Upper and lower endoscopy with multiple biopsies -radiographic imaging (small bowel NMR)

-Screen for extraintestinal manifestations of CD -laboratory tests

(consider serologic markers: pANCA, ASCA)

Typical picture
$\Rightarrow \begin{aligned} & \text { Crohn's disease activity index } \\ & \text { Quality of life evaluation } \\ & \text { treatment }\end{aligned}$

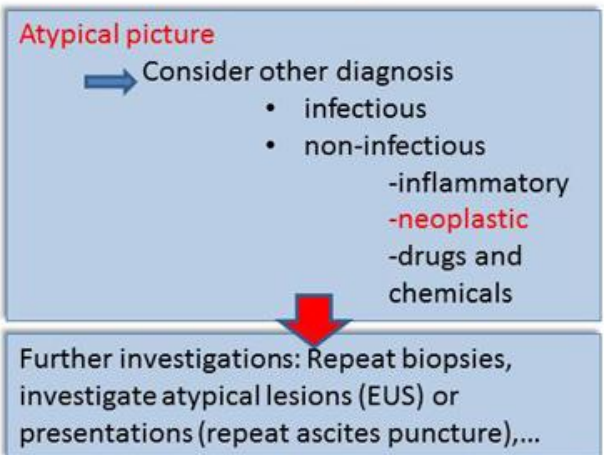

*In the first case, ascites, absence of diarrhea and atypical gastric lesions should have led to repeated biopsies, ascites fluid analysis and imaging investigations (e.g. EUS) to rule out malignancy.

*In the second case, enlarged gastric folds and predominant upper gastrointestinal tract symptoms should have led to EUS, repeated biopsies and CT or NMR imaging

Fig. 4. Diagnostic sequence in case of CD suspicion.

\section{References}

1 Loftus EV Jr, Schoenfeld P, Sandborn WJ: The epidemiology and natural history of Crohn's disease in population-based patient cohorts from North America: a systematic review. Aliment Pharmacol Ther 2002;16:51-60.

-2 Katon RM, Brendler SJ, Ireland K: Gastric linitis plastica with metastases to the colon: a mimic of Crohn's disease. J Clin Gastroenterol 1989;11:555-560.

-3 Tanakaya K, Takeuchi H, Yasui Y, Takeda A, Umeda Y, Murakami I: Metastatic carcinoma of the colon similar to Crohn's disease: a case report. Acta Med Okayama 2004;58:217-220.

4 Barros e Silva M, Paiva T Jr, Benevides L, Chinem L, Fanelli M: Age and peritoneal carcinomatosis in gastric cancer patients. J Clin Oncol 2008;26:15548.

5 Mayer R: Gastrointestinal tract cancer: tumors of the stomach; in Kasper D, Braunwald E, Fauci S, Hauser S, Longo D, Hameson J, Loscalzo J (eds): Harrison's Principles of Internal Medicine, ed 17. New York, McGraw-Hill Medical, 2008, pp 571-573.

6 Lee JU, Kim YH, Lee SY, Kim KH, Chung CH, Kim KH, Son HJ, Rhee PL, Kim JJ, Rhee JC: Do es ascites mean more severe Crohn's disease? Korean J Gastroenterol 2004;43:304-309.

-7 Ginès A, Pellisé M, Fernàndez-Esparrach G, et al: Endoscopic ultrasonography in patients with large gastric folds at endoscopy and biopsies negative for malignancy: predictors of malignant disease and clinical impact. Am J Gastroenterol 2006;100:64-69.

8 Ishigami H, Kaisaki S, Kitayama J: The updated treatment strategy for gastric cancer with peritoneal metastasis. Nihon Geka Gakkai Zasshi 2012;113:18-21.

9 Baumgart DC, Sandborn WJ: Inflammatory bowel disease: clinical aspects and established and evolving therapies. Lancet 2007;369:1641-1657. 
10 Lee HC, Yang MT, Lin KY, Tu HY, Zhang TA, Chen PH: Metastases from gastric carcinoma to colon in the form of multiple flat elevated lesions: a case report. Kaohsiung J Med Sci 2004;20:552-557.

11 Metayer P, Antonietti M, Oumrani M, Hemet J, Lemoine F, Basuyau J: Metastases of gastric adenocarcinoma presenting as multiple intestinal polyps. Dis Colon Rectum 1991;34:622-623.

12 Dohden K: Metastases from gastric carcinoma to esophagus, duodenum and large intestine in the form of polyposis, report of a case. Stomach Intestine (Tokyo) 2002;37:1238-1242.

13 Ogiwara H, Konno H, Kitayama Y, Kino I, Baba S: Metastases from gastric adenocarcinoma presenting as multiple colonic polyps. Surg Today 1994;24:473-475. 